\title{
Asymptotic level spacing distribution for a q-deformed random matrix ensemble
}

\author{
K A Muttalib and J R Klauder $†$ \\ Department of Physics, University of Florida, Gainesville, FL 32611, USA
}

Received 20 February 1996

Abstract. We obtain the asymptotic behaviour of the nearest-neighbour level spacing distribution for a $q$-deformed unitary random matrix ensemble.

The $q$-deformed unitary random matrix ensemble introduced in [1] describes a transition in spectral statistics from the highly correlated Gaussian unitary ensemble [2] (GUE, $q \rightarrow 1$ ) towards a completely uncorrelated Poisson ensemble $(q \rightarrow 0)$ as a function of $q$. Such transitions occur in a wide variety of physical systems including disorder and chaos [3]. In particular, the transition in the nearest-neighbour spacing distribution obtained from the model seems to show a remarkable similarity with that obtained numerically from a microscopic tight-binding Anderson model [4] describing the metal-insulator transition in disordered conductors. This similarity holds despite the fact that the problem of disordered conductors is known to have a small additional level-interaction $[5,6]$ which is not taken into account in [1]. (This term is known to change the numerical value of the variance of conductance by a very small amount in quasi-one dimension.) The similarity is all the more remarkable because it includes an apparent fixed point at $s=s_{0}$ in the spacing distribution $P(s)\left(s_{0} \simeq 1.8\right.$ for unitary ensembles and $s_{0} \simeq 2$ for orthogonal ensembles). The fixed point was used in [4] to propose the area $A=\int_{s}^{\infty} P(s) \mathrm{d} s$ as a one-parameter characterization of the spacing distribution. The length and disorder dependences as well as the scaling behaviour of the normalized parameter $\gamma=\left(A-A_{\mathrm{W}}\right) /\left(A_{\mathrm{P}}-A_{\mathrm{W}}\right)$, where the subscripts $W$ and $P$ refer to the Wigner and the Poisson limits, were studied in detail numerically and shown to contain information about the metal-insulator transition. However, the level spacing distribution of the $q$-ensemble requires the evaluation of a Fredholm determinant of the two-level kernel over a finite interval, and while the kernel was obtained analytically in [1], the determinant was evaluated numerically for various values of $q$. It is clearly of interest to evaluate analytically the spacing distribution as a function of $q$ in order to understand the role of $q$-deformation in these systems by, e.g., relating $q$ to the parameters $A$ and/or $\gamma$. While it has not been possible to obtain the complete spacing distribution analytically for all $s$ even in the GUE limit [2], the work of [4] shows that because of the (apparent) fixed point, $P\left(s \geqslant s_{0}\right)$ already contains valuable information. In the present work we obtain the asymptotic behaviour of the spacing distribution both near GUE and Poisson limits using the Szegö theorem on the asymptotic behaviour of a related Toeplitz

$\dagger$ Also at the Department of Mathematics.

0305-4470/96/164853+05\$19.50 (c) 1996 IOP Publishing Ltd 
determinant. Unfortunately, the method is not accurate enough to obtain information about the (apparent) fixed point itself, which requires evaluation of the terms independent of $s$ as well. However, by exploiting the existence of at least an approximate fixed point, we are able to determine the leading $s$-independent (but $q$-dependent) terms and obtain a reasonably good analytical expression for the asymptotic spacing distribution for $s \geqslant s_{0}$ near the GUE as well as the Poisson limits; this can then be used to make at least qualitative connection with the numerically accessible parameters $A$ or $\gamma$. Moreover, a qualitative understanding of the $q$-deformation of the spacing distribution obtained from our results should provide a basis for a more careful analysis to understand the nature of the fixed point.

We will assume that the $q$-ensemble is characterized, in the properly scaled variables where the average spacing between adjacent levels is unity, by the two-level kernel obtained in [1]:

$$
K(\zeta-\eta ; q \leqslant 1) \approx \frac{\beta}{2 \pi} \frac{\sin [(\zeta-\eta) \pi]}{\sinh [(\zeta-\eta) \beta / 2]} \quad q=\mathrm{e}^{-\beta} .
$$

Although the above kernel is valid only in restricted regimes [1], it is in these regimes that the kernel is translationally invariant and seems to be model independent [7]. We will obtain the asymptotic level spacing distribution given by this kernel. For comparison, the corresponding kernel for a GUE is given by

$$
K(\zeta-\eta ; q=1)=\frac{\sin [(\zeta-\eta) \pi]}{(\zeta-\eta) \pi}
$$

which is universal, independent of any parameter. The asymptotic behaviour of the level spacing for this kernel has been obtained in a variety of ways $[8,9]$, although a complete expression valid for all spacing is not available.

Given the kernel $K$, the probability $E(t)$ that the interval $(-t, t)$ does not contain any level can be obtained as a Fredholm determinant of the integral equation [2]

$$
\int_{-t}^{t} K(\zeta-\eta) f(\eta) \mathrm{d} \eta=\lambda f(\zeta)
$$

Changing variables such that the range of the integral is $(-1,1)$, one gets

$$
E(t)=\operatorname{det}\left(1-K_{t}\right)
$$

where

$$
K_{t}(\zeta-\eta ; q) \approx \frac{\beta t}{2 \pi} \frac{\sin [(\zeta-\eta) \pi t]}{\sinh [(\zeta-\eta) \beta t / 2]} .
$$

The nearest-neighbour spacing distribution is then given by

$$
P(s)=\frac{\mathrm{d}^{2}}{\mathrm{~d} s^{2}} E(s) \quad s=2 t .
$$

We first note that the kernel $K_{t}$ can be written as the Fourier transform of the function

$$
\phi(k)=\frac{\sinh [b]}{\cosh [b k / \pi t]+\cosh [b]} \quad b=\frac{2 \pi^{2}}{\beta} .
$$

To exploit the connection with the Toeplitz determinant, consider the function

$$
f(\theta)=1-\frac{\sinh [b]}{\cosh [b \theta / a]+\cosh [b]} .
$$

Then in the limit $N \rightarrow \infty$ and $N a \rightarrow 2 \pi t \gg 1$, we can identify $\operatorname{det}\left[1-K_{t}\right]$ with the $N \times N$ Toeplitz determinant

$$
D_{N}(f)=\operatorname{det}\left[\frac{1}{2 \pi} \int_{-\pi}^{\pi} f(\theta) \mathrm{e}^{\mathrm{i}(j-k) \theta} \mathrm{d} \theta\right]_{j, k=1,2, \ldots, N} .
$$


The function $f(\theta)$ has the following qualitative features. In the limit $\beta \gg 2 \pi^{2}(b \ll 1)$, $f$ is a smooth non-zero positive function over the entire range $(-\pi, \pi)$. In the opposite limit $\beta \ll 2 \pi^{2}$, we can write

$$
f(\theta) \approx 1-\frac{1}{1+\exp ((b / a)(|\theta|-a))} .
$$

Note that the second term can be thought of as a Fermi function with temperature $a / b$ and chemical potential $a$. For $\beta \rightarrow 0, f(\theta)=0$ for $-a<\theta<a$, and 1 for $|\theta|>a$. For non-zero but small $\beta$, the function is still exponentially small within some finite range of $\theta$, and we can use the Fermi function analogy to approximate the function as zero for $|\theta|<\alpha$, where $\alpha \approx a(1-1 / b)$. We will see below that as long as $f(\theta)$ can be considered to be zero in some range of $\theta$, the asymptotic spacing distribution is 'GUE-like', with $P(s) \sim \exp \left(-\right.$ constant $\left.\times s^{2}\right)$. When there is no gap, i.e. $f(\theta)$ is non-zero everywhere, the asymptotic spacing distribution is 'Poisson-like', with $P(s) \sim \exp (-\operatorname{constant} \times s)$. The crossover occurs around $\beta \approx 2 \pi^{2}$.

We first consider the simpler limit $\beta \gg 2 \pi^{2}$. In this limit $f(\theta)$ is a smooth non-zero positive function over the entire range $(-\pi, \pi)$, and a theorem due to Szegö [10] allows us to write down the asymptotics

$$
\ln D_{N}(f) \approx N f_{0}+\frac{1}{4} \sum_{k=1}^{\infty} k f_{k} f_{-k}+\mathrm{O}(1)
$$

where $f_{k}$ are the Fourier coefficients of $\ln f(\theta)$ :

$$
\ln f(\theta)=\sum_{k=-\infty}^{\infty} f_{k} \mathrm{e}^{\mathrm{i} k \theta} .
$$

Consider the first term

$$
\begin{aligned}
f_{0} & =\frac{1}{2 \pi} \int_{-\pi}^{\pi} \ln f(\theta) \mathrm{d} \theta \\
& =\frac{a}{\pi b} \int_{0}^{b \pi / a} \ln [1-g(x)] \mathrm{d} x
\end{aligned}
$$

where

$$
g(x)=\frac{\sinh [b]}{\cosh [x]+\cosh [b]} .
$$

In the limit $N \rightarrow \infty$ and $N a \rightarrow 2 \pi t=\pi s$, we can take the upper limit to $\infty$, and obtain

$$
N f_{0}=\frac{s}{b} \int_{0}^{\infty} \ln [1-g(x)] \mathrm{d} x
$$

Note that this term is linear in $s$. The next term is independent of $N$. Since $N a \rightarrow \pi s$, it must be independent of $s$ as well. To leading order, it turns out to be independent of $a$, so the term is of $\mathrm{O}(1)$. Clearly it is $b$-dependent. However, note that the asymptotic formula is not very accurate in this order, so we will not gain much by evaluating this term. Instead, since we already have the correct asymptotic $s$ dependence, we will use the existence of a fixed point at $s=s_{0} \simeq 1.8$ to obtain the leading $s$-independent terms. Note that the fixed point is seen in the numerical evaluation of the determinant in [1]. We do not know to what accuracy the fixed point can be considered exact. We will assume that it can be considered exact at least up to leading order in $\beta$. 
Going back to the first term, expanding the logarithm inside the integral in (15) for small $b$, the leading term gives $-s$, the next term has the leading correction due to $\beta$, given by $-b / 6=-\pi^{2} / 3 \beta$. To leading order, we then have

$$
E(s ; \beta) \approx \exp \left[-s\left(1+\pi^{2} / 3 \beta\right)+C\right]
$$

where $C$ includes the $\beta$-dependent terms not calculated in this scheme. The spacing distribution is given by a second derivative of $E(s)$ with respect to $s$, and we obtain

$$
P(s) \approx \exp \left[-s\left(1+\pi^{2} / 3 \beta\right)+2 \pi^{2} / 3 \beta+C\right]
$$

where we have written the prefactor as the exponential of a logarithm and kept only the leading order terms. We now demand that there exists a fixed point at $s_{0}$ such that all curves of $P(s)$ as a function of $s$ for various values of $\beta$ cross through this point, at least up to leading order in $\beta$. The fixed point is where these curves meet the Poisson curve given by $P_{\mathrm{P}}(s)=\mathrm{e}^{-s}$. This means that the constant $C$ must be such that

$$
P(s) \approx \exp \left[-s-\left(s-s_{0}\right) \pi^{2} / 3 \beta\right]=P_{\mathrm{P}}(s) \mathrm{e}^{-\left(s-s_{0}\right) \pi^{2} / 3 \beta} \quad \beta \gg 2 \pi^{2} .
$$

The value of $s_{0}$ is given by the (larger) crossing point of the Poisson curve with the GUE curve, and turns out to be close to 1.8 . We can now use equation (18) to identify the parameter $\beta$ with those of [4]. The area under the curve from $s_{0}$ to $\infty$ is simply

$$
A=\frac{\mathrm{e}^{-s_{0}}}{1+\pi^{2} / 3 \beta} \text {. }
$$

Since $A_{\mathrm{P}}=\exp -s_{0}$, it is easy to see that the normalized scaling parameter $\gamma$ is given by $(1-\gamma) \propto 1 / \beta$.

We now consider the opposite limit, $\beta \ll 2 \pi^{2}$. In this limit as we mentioned before, the function $f(\theta)$ can be considered to be zero for $|\theta|<\alpha=a\left(1-\beta / 2 \pi^{2}\right)$. As soon as the function is zero for some range of $\theta$, the Szegö theorem we used above no longer applies, and we have to use a generalization of the theorem due to Widom [11]. The analogous formula for the asymptotic behaviour for the determinant reads

$\ln D_{N}(f) \approx N^{2} \ln \cos \frac{\alpha}{2}-\frac{1}{4} \ln \left(N \sin \frac{\alpha}{2}\right)+N F_{0}+\frac{1}{2} \sum_{k=1}^{\infty} k F_{k} F_{-k}+B$

where

$$
F(\theta)=f\left(2 \cos ^{-1} \cos \left(\frac{1}{2} \alpha\right) \cos \theta\right)
$$

and $B$ is a constant. $f$ is the same function defined as in (8). Taking the limit $N \rightarrow \infty$, $N a=\pi s \gg 1$ we get

$\ln D(f) \approx-\frac{1}{8}(\pi s)^{2}\left(1-\frac{\beta}{2 \pi^{2}}\right)^{2}-\frac{1}{4} \ln \left[\frac{\pi s}{2}\left(1-\frac{\beta}{2 \pi^{2}}\right)\right]+N F_{0}+\frac{1}{2} \sum_{k=1}^{\infty} k F_{k} F_{-k}+B$.

The term involving $F_{k}$ is independent of $N$ and therefore $s$; so in the spirit of our previous scheme we will not evaluate it. The only term left to consider is $N F_{0}$, where

$$
\begin{aligned}
F_{0} & =\frac{1}{2 \pi} \int_{-\pi}^{\pi} \ln F(\theta) \mathrm{d} \theta \\
& =\frac{1}{2 \pi} \int_{\alpha}^{2 \pi-\alpha} \ln f(\phi) \frac{\sin [\phi / 2]}{\sqrt{\cos ^{2}[\alpha / 2]-\cos ^{2}[\phi / 2]}} \mathrm{d} \phi
\end{aligned}
$$


where we have made the change of variables $\phi=2 \cos ^{-1} \cos \left(\frac{1}{2} \alpha\right) \cos \theta$ as given in (21). For $\beta \ll 2 \pi^{2}$, the function $f(\phi)$ is well approximated by equation (10), and its logarithm is appreciable only for $\phi \leqslant a$. In this regime the argument of the logarithm does not change much, and we can replace it by a constant $\frac{1}{2}$. The integral can then be easily evaluated giving

$$
N F_{0} \approx-\frac{\ln 2}{2 \pi} \sqrt{\beta} s
$$

Up to linear order in $\beta$, equation (22) then leads to

$$
E(s ; \beta) \approx E(s ; \beta=0) \exp \left[\frac{\pi}{8}\left(\sqrt{\beta} s-\frac{2 \ln 2}{\pi^{2}}\right)^{2}+B_{1}\right]
$$

where $B_{1}$ is independent of $s$ but includes $\beta$ dependent terms. The spacing distribution obtained from (6) in the asymptotic limit $\pi s \gg 1$ can now be approximately given by

$P(s) \approx P_{\mathrm{W}}(s) \exp \left(\frac{\pi}{8} \sqrt{\beta}\left(s-s_{0}\right)\left[\sqrt{\beta}\left(s+s_{0}\right)-2 \frac{2 \ln 2}{\pi^{2}}\right]\right) \quad \beta \ll 2 \pi^{2}$

where the constant $B_{1}$ in (25) has been fixed according to our previous scheme, namely by demanding the existence of a fixed point up to this order at $s=s_{0}$, and $P_{\mathrm{W}}(s)$ denotes the Wigner or the GUE spacing distribution.

Note that as long as $\sqrt{\beta} s_{0}>2\left(2 \ln 2 / \pi^{2}\right)$, the tail of the distribution beyond $s_{0}$ is above the limiting GUE tail, going towards the Poisson limit with increasing $\beta$, as expected. However, in the limit $\sqrt{\beta}\left(s+s_{0}\right)<2\left(2 \ln 2 / \pi^{2}\right)$, part of the tail of $P(s)$ beyond $s_{0}$ is below the GUE tail, going away from the Poisson limit with increasing $\beta$. This is counterintuitive. We suspect that the nature of the asymptotics, given by $\beta \rightarrow 0$ faster than $s \rightarrow \infty$, makes our approximation unreliable in this latter restricted regime.

Equations (18) and (26) are our main results. Detailed comparison with numerical results for transitions in spacing distribution seen in a variety of complex physical systems [3, 7] might elucidate any possible underlying connection between complexity and $q$-deformation. The results should also provide the basis for a more careful study of the unexpected fixed point.

\section{References}

[1] Muttalib K A, Chen Y, Ismail M E H and Nicopoulos V N 1993 Phys. Rev. Lett. 71471

[2] See, e.g.:

Mehta M L 1991 Random Matrices 2nd edn (New York: Academic)

[3] See, e.g.:

Giannoni M-J, Voros A and Zinn-Justin J 1991 Chaos and Quantum Physics (Les Houches LII) (Amsterdam: Elsevier)

Reichl L E 1992 The Transition to Chaos (Berlin: Springer)

[4] Shlovskii B I et al 1993 Phys. Rev. B 4711487

[5] Beenakker C W J and Rejaei B 1993 Phys. Rev. Lett. 713689

[6] Muttalib K A 1995 J. Phys. A: Math. Gen. 28 L159

[7] Blecken C, Chen Y and Muttalib K A 1994 J. Phys. A: Math. Gen. 27 L563

[8] des Cloizeaux J and Mehta M L 1973 J. Math. Phys. 141648

[9] Dyson F J 1976 Commun. Math. Phys. 47171

[10] Grenander U and Szegö G 1958 Toeplitz Forms and Their Applications (Berkeley, CA: University of California Press)

[11] Widom H 1971 Indiana Univ. Math. J. 21277 